\title{
Dermoscopy of frictional asymptomatic darkening of the extensor surfaces
}

\author{
Cheng Tan', Lulu Xia² \\ ${ }^{1}$ Department of Dermatology, Jiang-Ning District Hospital of Traditional Chinese Medicine, Nanjing, China \\ 2Department of Dermatology, Affiliated Hospital of Nanjing University of Chinese Medicine, Nanjing, China
}

Adv Dermatol Allergol 2019; XXXVI (2): 232-233

DOI: https://doi.org/10.5114/ada.2019.84598

Frictional asymptomatic darkening of the extensor surfaces (FADES) is an uncommon skin disorder. Clinically, it appears as symmetrical brownish patches on the extensor surface of the elbows [1]. Dermoscopically, little is known about it. We herein revealed some dermoscopic features on a FADES patient that might provide clues for its diagnosis.

A 46-year-old Chinese male was seen in May 2014, complaining of a seasonal variation of a brownish skin discoloration on his elbows. The lesion has tended to appear yearly in summer, and then alleviated and completely disappeared in winter for 10 years. He was otherwise in a good health, and denied any abnormal sensations and history of exposure to chemicals. There was a frequent friction of the elbow directly against the desk surface for everyday working reasons, especially in summer. On examination, velvet, brownish and hyperkeratotic lesions were distributed over the extensor surface of both elbows and extended to the neighbouring skin

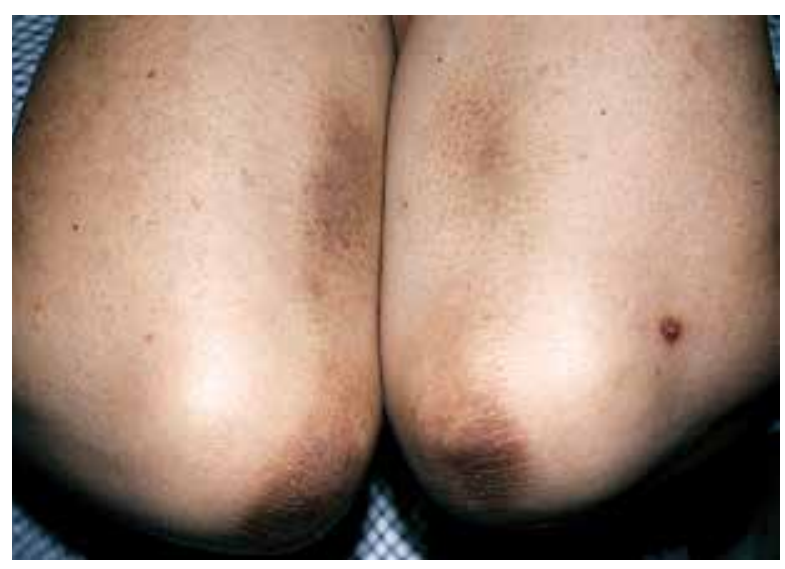

Figure 1. Velvet, brownish and hyperkeratotic patches were distributed over the extensor surface of both elbows and extended to the neighbouring skin area. Erythemas or scales were unnoticeable area. Erythemas or scales were unnoticeable (Figure 1). Other parts of the skin were normal. Clinical examination with dermoscopy was performed. Dermoscopic images from each lesion were obtained using a lens (Dermoscope Delta 20; Heine, Herrsching, Germany), mounted on a Cannon EOS 550 camera. Compared to the normal skin area, the lesion showed multiple, sparse brownish uniformly-sized clods in some parts of interconnecting white lines. Small white dots were surrounded by brownish clods (Figure 2). Skin biopsy revealed hyperkeratosis, hypergranulosis and pronounced epidermal invagination due to papillomatosis. There was a sparse infiltration of lymphocytes around superficial vessels (Figure 3). All these indicated the diagnosis of FADES. A 2-month application of $0.1 \%$ Tretinoin cream and avoidance of direct

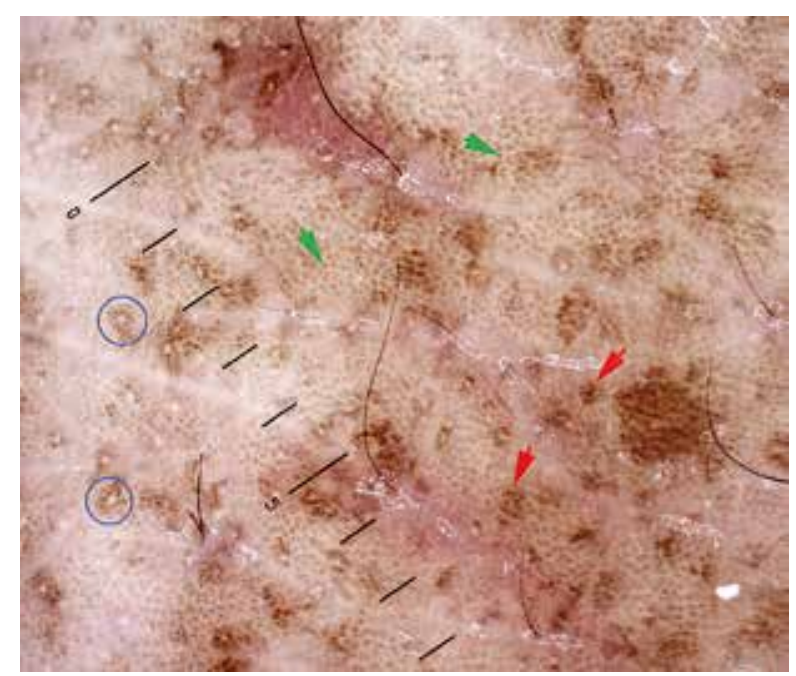

Figure 2. Dermoscopic examination of the lesion revealed multiple, sparse, brownish, and uniformly-sized clods (red arrows) in some parts of interconnecting white lines (green arrows). Besides, small white dots were seen with adjacent brownish clods (blue circles)

Address for correspondence: Cheng Tan MD, Department of Dermatology, Affiliated Hospital of Nanjing of Chinese Medicine, 155 Hanzhong Road, 210029 Nanjing, China, phone: +86 13515102233, e-mail: tancheng@yeah.net Received: 16.06.2016, accepted: 26.11.2017. 


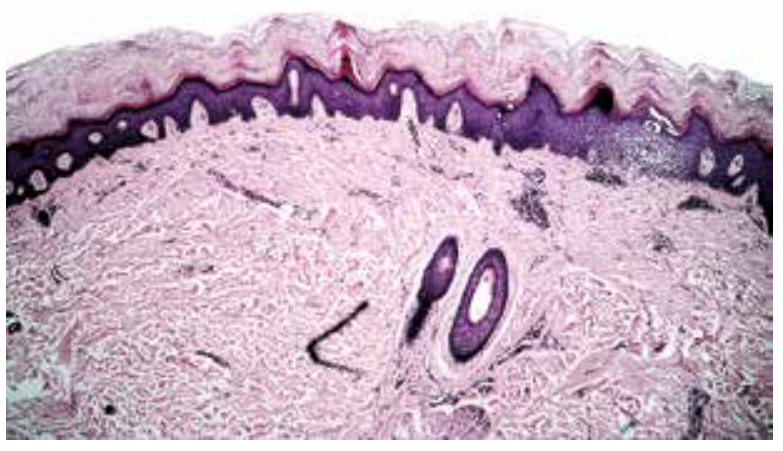

Figure 3. Skin biopsy revealed hyperkeratosis, hypergranulosis and pronounced epidermal invagination due to papillomatosis. There was a sparse infiltration of lymphocytes around superficial vessels (HE; 100x)

friction resulted in complete regression of the lesions much earlier before the onset of autumn that year, and did not relapse ever since.

The FADES is an uncommon frictional skin disorder that was first defined by Krishnamurthy et al. in 2005 [1]. It is characterized by a uniform, asymptomatic and brown darkening over the extensor surfaces of the elbows and knees [1-4]. However, similar cases had been reported previously. In 1954, Ber et al. described a form of hyperkeratinization of the elbows and knees which they ascribed it to the localized high pressure on it [5]. Shortly after that, Dahl [6] reported another patient with chronic pulmonary emphysema who similarly developed asymptomatic hyperpigmentation on the anterior distal thighs, which was a direct consequence of characteristic "dyspnoeic posture" (leaning forward, supporting the weight of the trunk by placing hands or elbows on thighs just above the knees). Krishnamurthy et al. believed that a localized friction attributed to the pathogenesis of the above cases. Our case was inconsistent with these clinical features. Pathologic findings of FADES may consist of hyperkeratosis, acanthosis, papillomatosis, with minimal underlying inflammation [1].

Dermoscopy is a well-established diagnostic tool to improve the clinical recognition of a broad spectrum of skin disorders. Up to now, no dermoscopic inspection has been recorded in FADES probably due to limited reported cases. Dermoscopically, in this case, we observed multiple, sparse, brownish, and uniformly-sized clods in some parts of interconnecting white lines. Besides, small white dots were seen with surrounding brownish clods.

We believe the above dermoscopic clues may aid the diagnosis of FADES; however, its potential diagnostic value should be confirmed in a larger group of patients.

\section{Acknowledgments}

The study was financially supported in part by the National Natural Science Foundation of China (Grant No. 81173400).

\section{Conflict of interest}

The authors declare no conflict of interest.

\section{References}

1. Krishnamurthy S, Sigdel S, Brodell RT. Frictional asymptomatic darkening of the extensor surfaces. Cutis 2005; 75: 349-55.

2. Wahlberg JE. Occupational hyperkeratoses in carpet installers. Am J Ind Med 1985; 8: 351-3.

3. Menne T, Hjorth N. Frictional contact dermatitis. Am J Ind Med 1985; 8: 401-2.

4. Menne T. Frictional dermatitis in post-office workers. Contact Dermatitis 1983; 9: 172-3.

5. Ber A. The sign of dirty knees and elbows. Acta Endocrinol (Copenh) 1954; 16: 305-8.

6. Dahl MV. Emphysema. Arch Dermatol 1970; 101: 117. 